\title{
Action professionnelle et pensée transformation
}

\begin{abstract}
Abstrait
Une visée d'action est toujours une visée de transformation du monde. En même temps, aux différentes étapes de son développement une action est toujours en transformation. Engager une recherche ou une pensée à propos de l'action en cours ajoute une transformation complémentaire. Ce texte décrit les différentes transformations co-présentes au sein de l'action, et leurs conséquences sur l'usage de la transformation comme paradigme pour penser les différentes actions dans lesquelles se trouvent simultanément engagés des acteurs professionnels et sociaux.
\end{abstract}

Mots-clés : action, transformation, pensée.

\section{Professional Action and "Transformation Thinking”}

\begin{abstract}
The goal of action is always the goal of transformation of the world. At the same time, at the different stages of its development, an action is always in transformation. Undertaking research or thinking about the action in progress adds a complementary transformation. This text describes the different transformations co-present within the action, and their consequences for the use of transformation as a paradigm for thinking about the different actions in which professional and social actors are simultaneously engaged.
\end{abstract}

Keywords: action, transformation, thinking.

\footnotetext{
* Conservatoire National des Arts et Métiers et Chaire Unesco - ICP, Paris, France. Article soumis le 2 février 2021 ; accepté pour publication le 24 février 2021.
} 


\section{Une redoutable obligation : professionnaliser par l'enseignement supérieur}

Le phénomène a pris une dimension internationale : la pression sociale qui s'exerce sur l'enseignement supérieur ne consiste pas seulement à lui demander de préparer les étudiants aux pré-requis de l'action professionnelle, elle lui demande également une préparation directe à l'action professionnelle elle-même, considérée comme déterminante aussi bien de l'efficacité productive que de l'emploi, et par là de la production des moyens d'existence.

Et c'est là bien sûr que les difficultés commencent : si les filières professionnelles ont pris progressivement une place essentielle dans l'enseignement supérieur et constituent depuis longtemps un outil de leur valorisation (ex. MIT : Massachusetts Institute of Technology), c'est au prix de multiples tensions entre formation et recherche, et entre les différentes formes de la recherche.

Les dispositifs de formation professionnelle supérieure mêlent souvent :

- des logiques disciplinaires traditionnelles, centrées sur la connaissance des entités du monde censées être les objets spécifiques des actions professionnelles, et sur la connaissance des situations d'exercice des actions professionnelles,

- des disciplines prescriptives centrées sur la logique d'intention et sur le résultat visé de l'acte professionnel spécialisé, traité le plus souvent selon une logique de succession temporelle,

- des formes de recherche centrées davantage sur l'optimisation de l'action professionnelle que sur son intelligibilité,

- le tout donnant lieu souvent à objectivation sous le terme de 'sciences et techniques' d'un champ de pratiques.

Beaucoup de formations professionnelles entretiennent ainsi une relation ambiguë avec le couple enseignement/recherche pourtant jugé caractéristique de l'enseignement supérieur depuis Humboldt, et fondé sur le triptyque production de $\underline{\text { savoirs par la recherche/diffusion de savoirs par l'enseignement/application de }}$ savoirs par l'action.

Ce triptyque constitue la référence obligée à la fois des modes de gestion des profils des personnels de l'enseignement supérieur (enseignants/chercheurs), de l'organisation de la formation (enseignements magistraux/pratiques) et de l'organisation de la recherche (recherche fondamentale/appliquée).

Et on ne doit pas s'étonner dans ces conditions qu'émergent de nouvelles organisations inversant ce triptyque, mettant en priorité l'expérience, puis la recherche sur l'action professionnelle y compris par les acteurs professionnels euxmêmes, et $\mathrm{y}$ associant la formation, favorisant de ce point de vue des profils de triple compétence professionnel/chercheur/formateur.

Ces nouvelles formes d'expérience, de recherche et de formation obligent à se poser comme question de recherche une question vieille comme la réflexion sur l'organisation de l'action : que produit l'action professionnelle? 
Qu'est-ce que l'action professionnelle change au monde ? Dans quelles conditions elle le change ? Comment peut-on analyser (et non pas évaluer) l'action professionnelle? Quelle portée peut-on donner à la production de savoirs relatifs à l'action?

\section{Une question particulièrement sensible dans les métiers de l'humain}

Cette question se pose avec une acuité particulière dans le domaine des actions professionnelles qui consistent à 'changer quelque chose' chez d'autres sujets, et à être socialement mandaté pour le faire ; on tend alors à parler alors de métiers de l'humain : par exemple éducation, travail social, santé, communication, management.

Tous ces métiers consistent à 'agir sur autrui' ; on peut les appeler aussi 'métiers de la société'. Ils constituent une sorte de travail de la société sur ellemême.

\section{Agir sur autrui, c'est agir sur l'activité d'autrui}

Tous les métiers de l'humain ont une même visée principale : ils ont pour ambition d'agir sur autrui en agissant sur l'activité d'autrui. Et ils attribuent à cette fin leurs publics des caractéristiques légitimant leur action professionnelle ; ils les 'étiquettent' conformément au mandat social reçu.

Dans l'éducation, c'est par exemple l'étendue et la qualité des ressources dont disposent à titre personnel les apprenants (on parle quelquefois de 'biens intérieurs') et qu'ils sont susceptibles d'investir dans leur action qui sont situées comme 'objet' d'intervention. Ce n'est pas un hasard si dans la langue française on parle d"élèves' et si les programmes d'activités sont décrits notamment en termes de connaissances, de savoirs, de capacités, de compétences 'transmises'. Le discours d'analyse des activités éducatives, centré sur la fonction qu'elles jouent auprès des sujets concernés, peut les considérer comme des intentions de transformation d'habitudes d'activité chez les sujets apprenants.

Dans le travail social ce sont les personnes vulnérables que le discours social affiche comme objet principal d'intervention. Mais le discours d'analyse de ces activités, centré sur les fonctions visées auprès des personnes concernées peut aussi y voir une visée de transformation des rapports qu'elles entretiennent avec leur environnement social.

Les soins, au sens de 'cure', tendent à poser les problèmes en termes de souffrance ('patients' vient du latin 'patior' = souffrir), d'où le terme de guérison ; mais ils peuvent être tout aussi bien analysés comme visant des transformations des régimes d'activité des sujets concernés. Comme l'explique Georges Canguilhelm (1966), être malade c'est 'vivre d'une autre vie'. 
Dans la pratique de la communication, c'est la détention et la diffusion de l'information qui semble l'objectif social affiché ; mais du point de vue de l'analyse d'activités la communication peut être vue comme un couplage d'une activité d'offre de signification de la part du sujet énonciateur et d'une activité de construction de sens de la part du sujet destinataire, et donc comme un processus d'influence.

Dans le domaine du management : ce qui semble être le problème principal est le pilotage et l'utilisation optimale des moyens matériels et humains de l'action, alors que du point de vue de l'analyse des activités on peut voir aussi les activités managériales comme une intervention sur l'engagement d'activité d'autrui (Barbier 2017 : 533-551).

Que l'on soit donc du point de vue du discours social ou du point de vue de l'analyse des activités centrée sur la fonction jouée auprès des personnes visées, il ne semble pas qu'on ne puisse pas échapper à ce paradigme de pensée : l'action professionnelle a toujours une visée de transformation.

\section{Agir sur l'activité (ibidem) : un 'couplage' entre l'activité d'un sujet intervenant et l'activité d'un sujet visé par l'intervention}

Tous les métiers et activités relevant du champ de l'humain sont identifiables par l'organisation sociale d'interactions ou par l'émergence d'interactivités ayant une incidence sur les transformations d'autrui.

Les interactions peuvent désigner les activités qui intentionnellement organisent ces transformations, alors que les interactivités pourraient désigner plutôt les activités qui surviennent du seul fait de la co-présence de plusieurs sujets dans une situation, et qui auraient la même incidence sans forcément une organisation intentionnelle. Non seulement les transformations qui en résultent ne se limitent pas aux transformations intentionnelles, mais, de plus, elles touchent tout autant les sujets intervenants que les sujets visés, même si les intervenants l'ignorent. Les uns et les autres se transforment solidairement.

Les interactions intentionnelles fonctionnent comme des ouvertures à autrui d'espaces d'activité, ce qui explique la fréquence du terme 'dispositifs', ou 'propositions d'activités'. Le phénomène est particulièrement visible par exemple dans le domaine du 'handicap' où l'essentiel du travail de l'éducateur consiste à imaginer des activités susceptibles de provoquer l'intérêt des sujets pour entrer dans l'activité ou pour l'infléchir (Dutoit 2018).

Cette ouverture à l'activité d'autrui est présente dans tous les métiers de l'humain : - Organisation d'activités susceptibles d'engendrer des apprentissages dans le cas de l'éducation et de la formation : entraînements, études de cas, exercices - Organisation de situations de production réelle ou de responsabilité professionnelle dans le cas du développement de compétences et de la professionnalisation - Offre de significations adressées à des destinataires, par le 
discours, par la démonstration ou par l'exemple dans les situations de communication. - Organisation de situations de surprise ou d'étonnement dans les dispositifs de constitution d'expérience. - Organisation de nouvelles situations pour l'engagement des activités des « collaborateurs » ou des salariés dans le cas de réorganisations d'entreprises ou suscitées par des dirigeants.

Toutes les situations d'intervention sur l'activité d'autrui peuvent ainsi être lues ainsi comme des ouvertures d'espaces d'activités, comme des propositions d'activités à autrui. Ainsi le cours magistral, exemple emblématique d'activités discursives manifestes de la part du professeur, et situation apparemment dénuée de réciprocité de la part de l'auditeur, peut se révéler au contraire une invitation à un flux considérable d'activités mentales, éventuellement inédites, de la part de l'auditeur.

Les activités d'intervention sur l'activité d'autrui n'ont certes pas pour objectif de transformer l'activité des sujets intervenants ; mais en réalité ils la transforment considérablement et notamment en ce qui concerne l'élaboration de leurs propositions d'activités : c'est sur ce terrain qu'ils se constituent une 'expérience propre' d'intervenant.

L'influence sur l'activité d'autrui ne s'opère toutefois que si l'espace ainsi ouvert est investi par les sujets visés, qui le transforment dans un autre espace propre de sujet impliqué dans sa propre transformation. Pas d'éducation bien sûr sans activité propre à effet d'apprentissage de la part de l'apprenant, pas de soin sans 'travail' du malade, pas de management sans 'implication' du collaborateur. Toutes les interventions sur l'activité d'autrui supposent un investissement effectif par les sujets visés des espaces d'activités ouverts.

Les interventions ne peuvent fonctionner que si elles suscitent donc la performation de l'activité des sujets visés par l'intervention, ce qui suppose que cette activité prenne sens pour eux. Ce sont ces performations d'activités, les affects qui les rythment, qui génèrent l'activité et quelle régénère. En retour, les sens construits par ces sujets peuvent contribuer aux transformations-objets de l'intervention : selon les cas changements d'habitudes d'activité, changements de régime d'activité, changements d'engagement d'activités, changements de rapports entre sujets et environnement social, etc.

\section{Agir sur l'activité autrui, c'est agir sur une dynamique préalable d'activité du sujet visé par l'intervention}

Les actions sur autrui, comme toutes les actions humaines, constituent des interventions sur des processus déjà en cours. Les ergonomes, dont la spécificité intentionnelle est d'agir sur le travail ont quelquefois l'habitude de décrire le travail comme une modification du 'cours naturel' des choses. La formule est discutable, mais elle a une éminente vertu : faire apparaître qu'action ou pas, un processus, une dynamique auraient de toute façon eu lieu, celle du sujet visé par l'action. Une 
situation d'action peut donc être considérée comme une articulation de plusieurs dynamiques, celle du sujet intervenant et celle du sujet visé. Généralement d'ailleurs, l'intervention ne s'effectue pas sur un seul processus, mais sur plusieurs, sur une configuration de processus.

Comme le dit à l'occasion le français Philippe Meirieu dans le domaine de l'éducation enseigner c'est au mieux favoriser les apprentissages, au pire ne pas les empêcher. Ceci explique l'importance qui s'attache, dans les métiers de l'humain, à identifier les dynamiques préalables d'activité, que cette identification donne lieu à opération explicite, ou qu'elle soit le produit d'une expérience, d'une familiarité préalable.

Une intervention sur autrui ne transforme pas directement au sens strict l'activité du sujet qu'elle vise. Elle n'élimine pas complétement l'activité manifeste du sujet qui peut constituer le point de départ de cette intervention (une erreur, un comportement inadapté ou dangereux, etc.). Elle s'inscrit dans et est susceptible d'infléchir l'activité déjà en cours du sujet visé par l'intervention.

Action éducative ou pas, soin ou pas, action sociale ou pas, communication ou pas, les sujets se construisent du simple fait de leurs activités. S'il peut arriver que ce soient les activités manifestes, socialisées, d'un sujet qui constituent le point de départ d'une intervention, ces activités manifestes ne sont en fait que des actualisations, situées dans un temps, dans un espace, et dans des circonstances données, de l'activité de ce même sujet, entendue comme son potentiel de transformation de son environnement et de lui-même.

Au-delà des activités constatées, se trouvent en jeu des activités empêchées (Clot 2006) des activités refoulées, des activités simulées, etc. ... ; bref, d'une manière générale, les possibles d'activité de ce même sujet tels que construits dans et par son histoire antérieure. Nous appelons dynamiques d'activité les régularités repérables dans le développement de cette activité. Ce sont elles qui sont l'objet réel de l'intervention. Ce sont elles qui peuvent se trouver infléchies à la suite de l'intervention.

Ceci peut conduire par exemple les intervenants à repérer avant même l'engagement de leur propre action les dynamiques de changement déjà présentes chez les sujets visés lors de la première demande d'aide (Martine Dutoit dans ce numéro).

\section{Agir sur l'activité d'autrui c'est construire une situation d'action}

Les situations d'action sont, dans l'intervention, des situations construites; elles sont de fait le produit d'interprétations de la part des acteurs impliqués, et notamment de l'acteur qui engage l'action. Ceux-ci ne se contentent pas de prendre connaissance de la situation, ils la construisent ; elles ne sont donc pas seulement données, elles sont aussi fabriquées. Elles ne sont donc pas seulement l'objet de 
moments de réflexion, elles en sont aussi le produit (Myriam Léonard, dans ce numéro).

Ces constructions mettent en jeu les cadres mentaux préalablement constitués, tels qu'issus notamment de l'expérience sociale des participants. Ce qui semble jouer un rôle majeur peut être défini comme l'expérience qu'ils ont des autres acteurs agissants, ce qui pourrait se résumer de la façon suivante : non pas penser l'autre, mais penser comment pense et agit l'autre. La construction de l'expérience d'autrui peut certes s'effectuer en utilisant des cadres interprétatifs issus de savoirs formels, mais elle s'effectue le plus souvent à travers des phénomènes tels que le vécu d'expériences semblables, de complicité ou d'identification aux personnes, phénomènes quelquefois désignés en termes d'empathie ou d'entrée dans la subjectivité d'autrui. Ces phénomènes s'observent dans tous les métiers de l'humain : influence sur l'activité des professeurs de leur propre expérience d'élève, influence sur l'activité des dirigeants de leurs trajectoires personnelles, influence sur l'activité des communicateurs de la diversité de leurs expériences de contacts sociaux.

La construction du destinataire de la communication par exemple, bien décrite en sciences du langage n'est qu'une modalité parmi d'autres, propre à la communication, de ce phénomène de construction. Pas de communication sans représentation de la culture de l'autre. On pourrait dire que les paroles fonctionnent comme l'offre de cadeaux : elles s'accompagnent d'anticipations sur la réaction des destinataires.

Ces constructions ont pour objet les sujets visés par l'action mais aussi en parallèle les figures réciproques des intervenants. De telles constructions concernent toutes les activités et tous les métiers de l'humain. Les acteurs, en particulier ceux qui engagent l'action, se font une représentation de l'environnement de l'action au regard de ce qu'ils ont à y faire, d'eux-mêmes et des autres comme sujets agissants dans la situation. Pour Max Pagès (1977) « une situation est l'ensemble des rapports concrets d'un sujet avec d'autres. La situation ne correspond à aucune définition absolue et a priori de ces rapports. Elle est l'ensemble des contradictions vécues du sujet avec d'autres » (ibidem 1977 : 9).

On remarquera la correspondance des outils susceptibles d'analyser ces phénomènes avec les outils utilisés dans l'analyse plus large des actions humaines, et dépassant les métiers de l'humain : image opérative de Dimitri Ochanine (Weill-Fassina 2016), sélective, déformée fonctionnelle, produite dans l'action, concept de représentamen chez Jacques Theureau (2010). Pour Alain Berthoz (2008), l'action est déjà dans la perception.

Dans certaines situations comme les situations de préparation explicite à l'action, on constate une transformation délibérée de ces constructions avant l'engagement de l'action : transformations de perception de la situation, activités de simulation, production de nouveaux possibles d'activité (thèse de Jasmine Hyppolyte dans ce numéro). 


\section{Agir sur l'activité d'autrui c'est éventuellement aussi transformer en cours d'action sa conduite}

Les transformations touchant l'action peuvent être constatables au cours même de la conduite de l'action. La conduite de l'action est constituée de l'ensemble des constructions mentales/affects survenant chez les sujets qui sont impliqués dans cette action, relatives aux organisations d'activité qui la caractérisent, lui confèrent un sens et sont susceptibles d'influer sur sa performation.

On tend alors à parler de ruptures, de conversions, d'inflexions. De telles ruptures sont repérables au niveau d'actions collectives, mais elles sont plus souvent identifiables au niveau individuel, notamment à travers les approches biographiques. L'empan temporel d'appréciation de telles transformations est alors souvent un temps long.

L'identification de telles transformations suppose une investigation des événements marquants, des expériences significatives décelables à travers des émotions longues et d'événements soudains réinitialisant les processus de construction de sens que les sujets confèrent à leur action individuelle/collective. On peut alors parler de reconstructions de sens (Thèse de Daniela Rodriguez dans ce numéro) ou de reconstruction des manières d'agir sur autrui et de faire agir autrui (Thèse de Kim Vu dans ce numéro).

Les émotions sont alors définies comme des ruptures/suspensions de l'activité en cours survenant conjointement à des reconstructions de sens que les sujets opèrent autour de leur propre action, et d'ouverture de nouvelles actions ou d'actions modifiées.

\section{Agir sur l'activité d'autrui c'est encore s'attendre à distinguer transformations intentionnelles et transformation effectives (thèse d'Izabella Kaminska dans ce numéro)}

Dans une expression célèbre, Freud en 1937 considère trois métiers d'intervention sur l'activité d'autrui, les plus manifestes à son époque (éduquer, gouverner, soigner), comme des professions 'impossibles'. « Il semble, écrit-il, que la psychanalyse soit la troisième de ces professions 'impossibles' où l'on peut d'avance être sûr d'échouer, les deux autres, depuis bien plus longtemps connues, étant l'art d'éduquer et l'art de gouverner (Freud (1985 [1937] : 263)».

On ne peut mieux introduire la distinction entre objectifs/résultats des actions et point de départ/résultante. Les résultats sont des évaluations des transformations opérées au regard des objectifs des actions, les résultantes sont l'état final de l'objet visé de leur intervention. Dans les métiers de l'humain, les intervenants 'ne savent pas ce qu'ils font' ; ils sont confrontés à l'inattendu, à l'incertain, à la fois à l'effet vertueux et/ou au contraire pervers de leur intervention. 
Les sujets engagés dans les métiers de l'humain peuvent se représenter ou produire des énoncés sur les transformations qu'ils souhaitent opérer chez les sujets visés par leur action, ils ne connaissent pas pour autant le point de départ précis de leur intervention, et partant ils ignorent largement les transformations qui s'opèrent effectivement, lesquelles dépassent singulièrement les résultats de l'évaluation qui peut en être faite. Si ces professionnels ont une claire connaissance des moyens qu'ils mettent en œuvre, ils reconnaissent souvent ne pas bien savoir ce qu'ils font au bout du compte.

Les actions d'intervention sur l'activité d'autrui sont des actions intention-

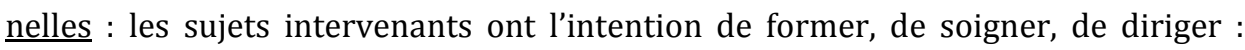
autre chose est de savoir ce que deviennent effectivement les sujets visés au terme de l'intervention. Les métiers de l'intervention sont aujourd'hui en plein développement (Barbier, Durand 2017) : si l'analyse de ce développement est traitée uniquement dans le cadre professionnel, le risque est assez fort de confondre les cultures professionnelles, les lexiques de l'action, centrés sur l'intentionnel, et cultures scientifiques, les concepts d'intelligibilité centrés spécifier les champs de pratiques par leur fonctions auprès des acteurs concernés, au-delà des intentions qu'ils affichent.

A minima, il convient donc de tirer, tant sur le plan de la recherche que sur le plan de l'action toutes les conséquences de la distinction possible entre actions et activités. Les actions sont des organisations singulières d'activités ordonnées autour d'une transformation du monde présentant une unité de fonction, de sens et de signification pour les sujets qui y sont engagés et leurs partenaires. L'activité est un processus de perception/transformation du monde et de perception/transformation de soi transformant le monde dans lequel et par lequel est engagé un être vivant dans ses rapports avec son environnement. Les actions s'intéressent aux transformations recherchées, les activités permettent d'accéder aux transformations effectives.

'Ce que nous sommes' est le produit de nos activités, alors que nos actions reflètent en bonne partie ce que nous voulons être. A partir des unes et des autres on peut se décrire ce que selon les cas on désigne comme individuation (Simondon 2007 ; Fleury 2018), de personnalisation (Tap 1987), d'anthroponomie (Bertaux 2015 ; Lojkine 2018).

\section{Engager une recherche sur son agir professionnel c'est aussi se transformer}

Au modèle production de savoir/transmission de savoir/application de savoir, qui reste largement dominant, s'est donc ajouté, notamment à partir des années 80 , un modèle liant toujours trois espaces, mais selon un mode nouveau, tendant cette fois à articuler explicitement action/production de savoirs/construction des sujets humains. 
Ce nouveau modèle n'émerge pas tout à fait des mêmes lieux : il est porté notamment des professionnels, des acteurs économiques, politiques et sociaux, des personnalités caractérisées par leur mobilité entre espaces professionnels et espaces universitaires.

Il présente également trois caractéristiques. Les recherches ont aussi pour objectif la connaissance des processus de transformation du monde, et notamment des interventions humaines. Ceci explique le développement de champs de recherche correspondant à des champs de pratiques : éducation, gestion, santé, travail social, sciences de l'ingénieur, etc. Elles peuvent se développer dans les espaces et dans les temps des actions qu'elles ont comme objet, avec leurs acteurs, et pour partie leurs méthodes ; bref, elles accompagnent les processus de transformation du monde. Les savoirs qu'elles produisent portent sur des actions singulières, situées ; ils sont immédiatement ré-investissables dans l'action par ceux qui les produisent.

Plusieurs courants déclinent actuellement ces caractéristiques. Les uns insistent sur l'indifférenciation des processus de recherche et des processus de transformation du monde : recherche-action, recherche-intervention, recherchedéveloppement, recherche professionnelle ; d'autres insistent sur les liens entre acteurs : recherche coopérative, recherche impliquée, recherche partenariale, collaborations praticiens-chercheurs ; d'autres enfin insistent sur le caractère singulier, situé de ses résultats : recherche clinique, recherche qualitative, recherche située, considérées comme plus proches des réalités connues des acteurs. Ces courants font appel à la fois à l'expérience et à la recherche.

La raison en est que les actions humaines sont dotées d'unités de sens et/ou de significations par les sujets qui y sont engagés. Elles s'accompagnent donc d'activités mentales relatives à leur propre développement. Ces activités mentales portent tantôt sur des existants (représentations finalisées) tantôt sur des souhaitables (représentations finalisantes). En même temps qu'ils agissent, les sujets développent donc notamment une activité continue de reconnaissance, de compréhension et d'interprétation du monde, qui met en jeu un grand nombre de constructions mentales antérieures.

Toutes ces activités mentales sont souvent socialement désignées comme connaissances (au pluriel) impliquant des constructions mentales antérieures. Les connaissances se transforment dans l'action. Se développent alors notamment des processus d'enquête au sens de Peirce et de Dewey, qui consistent à passer d'une situation indéterminée pour les sujets à une situation déterminée. A cette occasion se produit une reconstruction de leurs cadres mentaux, vécue comme un apprentissage. Les connaissances s'inscrivent ainsi plus généralement dans l'histoire des sujets censés les détenir. Elles sont à la fois des représentations du monde et dans le moment de leur activation des auto-représentations de soi se représentant le monde. 
La production de connaissances par les acteurs sur leurs propres actions et situations d'action est une occasion de formation et de développement. On parle notamment de formation par la recherche. Le développement de la pensée à partir de l'action favorise à l'évidence chez ceux qui la pratiquent l'exercice d'activités discursives sur leurs propres activités et des activités mentales qui y sont associées. Elle produit donc des compétences de rhétorique et de gestion de sa propre action, à ne pas confondre d'ailleurs avec des compétences d'action proprement dites. D’ailleurs, on constate, en dépit de velléités répétées, que les connaissances produites à cette occasion ne font guère l'objet de conservation, ou de nouvelles exploitations. C'est la raison pour laquelle nous pensons que leur fonction dominante est une fonction de professionnalisation et plus largement d'influence sur la construction des sujets professionnels et sociaux. Il en va de même des recherches-interventions sur les organisations qui influent sur la transformation de 'compétence collectives' de ces organisations.

Bien que la construction de connaissances dans ces conditions puisse être décrite souvent en termes de recherche au sens large (être en recherche), elle appartient en dominante au monde des apprentissages et plus généralement de la construction des sujets. La production de connaissances dans ces conditions est en effet à distinguer de l'activité sociale de recherche produisant et conservant des savoirs formalisés. La recherche académique est en effet une action ordonnée autour de savoirs inédits et d'une communication autour de ces savoirs dans des conditions permettant un jugement de valeur sur leur validité par une communauté destinataire.

Tous ces phénomènes que nous avons analysés comme relevant des activités humaines ordinaires trouvent leur fondement dans la capacité des sujets à conduire plusieurs activités à la fois, à les associer, à garder trace dans ces associations de leurs activités antérieures. Ce sont bien les mêmes sujets qui sont présents dans toutes les activités, quelle que soit l'intention dominante de l'action. Les actions produisent des transformations beaucoup plus larges que celles qu'elles prétendent produire. Il y a loin des intentions aux résultats et effets. Même si elles sont engagées pour des intentions spécifiques les actions humaines produisent d'autres résultats et effets que ceux qu'elles sont censées produire.

\section{Penser l'action/l'activité comme transformation}

Ainsi, de multiples points de vue, les actions/activités humaines peuvent être abordées comme des transformations. Mais les penser comme transformations peut alors conduire à être confronté notamment à quatre types de problèmes : 


\section{La continuité/discontinuité des transformations}

= Entrer par le concept d'activité, tel que défini plus haut, permet de penser la continuité des transformations.

Un exemple nous est donné par ce qu'il est convenu d'appeler les déplacements fonctionnels. Prenons quelques exemples :

- Les activités de reconnaissance : elles peuvent être définies comme des liens effectués par les sujets entre la connaissance née d'une activité en cours et la connaissance née d'une activité antérieure. Reconnaître c'est juger qu'un objet actuellement présent nous est déjà connu antérieurement à quelque degré. La perception fonctionne comme une activité de reconnaissance : pour William James (2005) « la sorte de connaissance appelée perception (...) est le type de cas dans lequel l'esprit jouit d'une 'familiarité' (acquaintance) avec un objet présent » (ibidem : 65). Elle suppose la mobilisation de cadres perceptifs issus d'expériences préalables. L'activité produit/transforme les cadres perceptifs investis dans les activités ultérieures de perception.

- Les activités de conduite des actions : elles font également apparaître que ce qui est le produit des activités de détermination des objectifs devient le moyen des activités d'évaluation sous forme de référent. De façon plus générale les activités mentales rétrospectives et anticipatrices constitutives de l'expérience apparaissent sous la forme d'un cycle itératif (Barbier 1991), où les unes fonctionnent tour à tour comme le matériau ou le moyen des autres.

- Les activités de communication elles-mêmes peuvent également être vues comme des transformations continues d'offres de significations : un certain nombre de linguistes considèrent que chaque prise de parole peut être vue comme une reconstruction de significations sur et pour le monde.

Tous ces processus ont un point commun : ils fonctionnent comme un

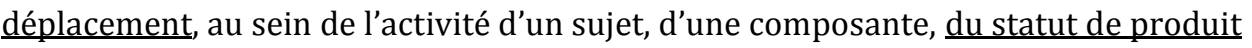
d'une activité antérieure au statut de moyen d'une activité ultérieure. Nous parlerons de déplacement fonctionnel, ou de mobilité fonctionnelle. Cette désignation est probablement proche du concept de migration systématique que l'on trouve chez Lev S. Vygotsky (Brossard 2004) à propos du développement de la vie émotionnelle. Ce concept a été repris pour parler d'autres formes de déplacement, notamment sous le vocable de migration fonctionnelle utilisé par Yves Clot (Dubosq, Clot 2010) et par des chercheurs se réclamant du courant de la clinique de l'activité.

Ces phénomènes de mobilité fonctionnelle jouent bien sûr un rôle majeur dans l'apprentissage et le développement des sujets individuels et collectifs. Ils jouent également un rôle important dans la définition que les sujets donnent des cadres de leur activité, c'est à dire du contexte qui la conditionne ou qui est censé la conditionner. Selon le mot de Philippe Astier (2003) l'activité de ce point de vue n'est pas seulement située, elle est situante. Le développement de l'activité est une incessante mobilité des composantes de l'activité. 
= Entrer par le concept d'action permet de penser leur discontinuité

Les actions se découpent en unités fonctionnelles du point de vue des acteurs qui les engagent. C'est une émotion d'acteur qui ouvre l'action et qui la délimite. Ce sont des préoccupations d'acteurs qui souvent permettent d'opérer des découpages dans l'analyse des interactions constitutives des métiers de l'humain. La notion d'événement, de sens, d'expérience significative n'a elle-même de portée que d'un point de vue d'acteur.

Par ailleurs il est aussi possible pour les actions, de les identifier à partir de la fonction qu'elles jouent auprès des acteurs concernés, à partir de ce qu'elles transforment-en-acte spécifiquement pour eux.

La perception peut ainsi être définie comme une transformation d'entités du monde en objets dotés d'unité pour l'activité dans laquelle est déjà engagé le sujet percevant. La représentation, comme l'activité permettant au sujet se représentant de rendre présent à lui-même et de façon différée des objets relevant d'autres espaces d'activité.

Différencier les actions par les transformations précises qu'elles opèrent est une entreprise difficile et exigeante mais qui peut avoir des effets heuristiques considérables. Elle permet par exemple de distinguer l'analyse et l'évaluation, régulièrement confondues. De façon plus générale cette entreprise exige de ne pas se satisfaire des mêmes outils pour aborder toutes les activités, mais au contraire de les spécifier et de les articuler dans le cadre de la construction d'une anthropologie plus générale des activités.

Le maître mot de l'analyse des actions est le concept d'unité fonctionnelle, pour un sujet ou un groupe de sujets. Elle est utilisée de fait dans les récits et en histoire.

\section{La poly-fonctionnalité des transformations}

On tend aujourd'hui à parler de complexité dès lors qu'on se trouve en présence d'un ensemble d'objets en interaction dynamique, s'auto-organisant, et formant de ce fait une unité. C'est le cas notamment du vivant. Le mot complexe présente toutefois l'inconvénient de caractériser à la fois un objet de pensée (on parle d'objet complexe) et la manière de le penser (on parle de pensée complexe).

Une analyse en termes de poly-fonctionnalité permet toutefois de rendre compte d'un certain nombre des phénomènes recouverts par le terme de complexité. C'est le cas par exemple de quatre types de transformations simultanées, c'est-à-dire de transformations survenant en même temps dans plusieurs espaces d'activités et affectant les sujets impliqués (Barbier, Durand 2017).

- Les transformations simultanées de l'activité et du sujet dans l'activité. L'expérience par exemple désigne à la fois les transformations de l'activité et du sujet-en-activité. 
- Les transformations simultanées de l'activité physique, de l'activité mentale, et des affects, comme c'est le cas dans l'émotion. L'émotion peut en effet être définie comme une rupture/suspension de l'activité en cours doublée d'une tendance même négative d'activité et d'une transformation des constructions de sens que les sujets opèrent autour d'elle.

- Les transformations simultanées de la représentation de l'activité et de la représentation de soi-en-activité comme le cas dans la conscience. La conscience peut en effet être définie comme un lien entre la représentation que le sujet se fait d'une activité et de lui-même comme sujet de cette activité.

- Les transformations simultanées de l'activité, du sujet en activité et du produit de l'activité, comme c'est le cas de la création. La création peut en effet être définie comme une action simultanée d'invention de soi, de transformation de son activité et de transformation du résultat de son activité, c'est-à-dire de production de l'œuvre.

Chacune des transformations coprésentes relève d'une analyse en termes de fonctionnalité, mais l'ensemble relève d'une analyse en termes de polyfonctionnalité. On observera que les expériences humaines jugées les plus significatives pour ceux qui les vivent peuvent se décrire en termes d'activités simultanées et relever d'une analyse poly-fonctionnelle.

\section{La solidarité des transformations des acteurs engagés conjointement dans l'action}

C'est la conséquence des couplages d'activité. Lorsque plusieurs acteurs sont impliqués dans une action chacun de ces acteurs se transforme du fait de cette participation. Même si ces transformations ne sont pas les mêmes, elles sont liées.

Une étude précise d'une action partagée (gravir un rocher) entre une professionnelle et un enfant handicapé (Dutoit, Barbier 2018) montre ainsi une transformation solidaire s'opérant chez les sujets en interaction ; on constate qu'en même temps que l'enfant se transforme dans son activité, la professionnelle se transforme également : ces transformations sont lisibles dans la manière dont se déroule le déplacement synchrone et son organisation. Ces transformations sont en lien et simultanées, mais ne sont pas identiques, et ne sont pas mentalisées, mais mises en acte. On peut accéder au 'dialogue corporel' par exemple à travers le souffle, le rythme cardiaque, les tensions des acteurs en train d'agir. Les corps distincts et joints portent et transmettent les informations sensorielles de l'un vers l'autre. Ils s'inter-informent de ce qui se passe pour l'un et pour l'autre dans le déroulement même de cette action partagée.

Les dispositifs d'intervention sur l'activité d'autrui n'ont pas pour objectif de transformer l'activité des professionnels intervenants ; en réalité ils la transforment considérablement. On tend à parler alors de professionnels 
expérimentés, qui peuvent d'ailleurs paradoxalement transférer dans leur fonction initiale l'expérience ainsi acquise. Ce n'est pas un hasard si les tuteurs qui acquièrent des compétences de communication sur le travail à l'occasion de leur activité de tuteur utilisent ces nouvelles compétences pour d'autres activités, de même si des dirigeants, au contact des dirigés, peuvent transformer leur manière de diriger, et être ainsi apparemment transformés par ceux-là mêmes qu'ils dirigent.

\section{Le passage d'un espace d'activité à un autre espace d'activité}

Les espaces d'activité se caractérisent, nous l'avons vu, par le statut de l'entité transformée (transformations mentales, transformations de significations, transformations de l'environnement). Le changement de statut des entités transformées peut s'opérer selon plusieurs modalités, comme par exemple :

- L'association qui est un lien de co-survenance entre plusieurs espaces d'activité dans lesquels se trouvent engagés en même temps le sujet. L'association émerge dans l'activité du sujet concerné. C'est le cas par exemple des activités d'évocation, qui associent des espaces mentaux et des espaces de communication, renvoyant éventuellement à des temporalités différentes.

- L'enchâssement qui peut être défini comme le développement, au sein de l'activité, d'une «boucle » à partir, sur et pour l'activité. C'est le cas en particulier de la réflexion en cours d'action ou de l'élaboration d'expérience, ou encore de l'enquête de John Dewey (1993). L'enchâssement implique une suspension de l'activité principale en cours.

L'investissement mutuel : lien pouvant être défini comme un investissement réciproque entre plusieurs espaces d'activités. Lev S. Vygotski (2019) a ainsi pu parler d'un investissement mutuel de la pensée et du langage, investissement qui apparaît particulièrement bien dans les activités de conceptualisation. Dans le cas des activités ayant donc pour intention d'agir sur l'activité (celle d'autrui ou la sienne), on pourra ainsi repérer systématiquement une transformation solidaire des activités et des sujets. L'accompagnement par exemple peut être considéré comme l'investissement mutuel d'une action d'optimisation et d'un espace de développement des acteurs. L'art peut être considéré comme l'investissement mutuel d'une activité d'expression et d'une activité de communication ; les figures littéraires (par exemple la métaphore) comme l'investissement d'espaces discursifs dans des espaces mentaux.

- La simulation : lien pouvant être défini comme la représentation d'un espace d'activité dans un autre espace d'activité, ou d'une action dans une autre action. C'est le cas par exemple des activités ludiques, des activités de conduite ou de préparation à l'action. 


\section{Penser transformation : Une exigence contemporaine}

Penser en termes de transformations fait l'objet aujourd'hui d'une demande sociale forte dans des champs divers.

\section{= Dans le champ des métiers sur les métiers :}

Formation, conseil, accompagnement, coaching, gestion des savoirs, sécurité, ingénierie, perfectionnement, développement des performances etc. Tous paraissent dominés aujourd'hui par les thématiques de la compétence de la professionnalisation et de la qualité. Si la notion de compétence peut être vue comme une inférence à partir d'une activité située, toujours en transformation, la professionnalisation elle, peut être considérée comme une transformation de compétences en rapport avec une transformation continue d'activités. Quant à la qualité, elle peut être analysée elle-même comme la transformation continue au sein de l'activité humaine, du rapport entre production et usage. Les métiers sur les métiers ont comme point commun de recourir à l’analyse des activités professionnelles avec/par/pour les sujets qui y sont engagés.

\section{= Dans le champ de la recherche}

Dans les sciences ayant pour objet le monde physique et le monde vivant on constate la montée du paradigme de l'émergence présenté comme alternatif à celui de la causalité pour rendre compte de phénomènes comme la vie, même si les définitions qui en sont données restent souvent dépendantes du référent causal : selon Malaterre (2010), l'émergence cherche à caractériser une impossibilité explicative à partir des propriétés et de l'arrangement des composants d'un système donné, autrement dit une impossibilité explicative à un niveau inférieur à celui du système en question. Il en va de même des phénomènes dits épigénétiques souvent décrits à la fois par référence et par différenciation avec le patrimoine génétique. Le paradigme de l'émergence invite en réalité à ne pas confondre phénomènes identifiés, et outils produits pour expliquer leur survenance. Il conduit aussi à interroger la pertinence de l'organisation de la recherche en disciplines, qui découpent leurs objets en fonction de leurs catégories d'analyse et recherchent souvent des explications à l'intérieur même de ces découpages. Dans les sciences qui prennent pour objet le monde social, on constate tout pareillement la montée du paradigme constructiviste, que l'on peut présenter comme une pensée de la transformation continue des activités et des sujets par et dans les activités. Ce paradigme invite à porter attention moins aux relations de causalité conditions/ événements/conséquences qu'aux corrélations de survenance entre phénomènes identifiés dans différents champs/espaces ; il invite également à relativiser les découpages disciplinaires au profit d'approches transversales. 


\section{= Dans les cultures d'action économique et sociale}

De façon plus générale, dans les cultures d'action économique et sociale contemporaines, on constate le passage d'une conception du progrès par spécialisation d'activités à une conception du progrès par recomposition. Dans tous les cas cette demande sociale exerce une pression à adopter une approche holiste des activités. Dans le cadre du développement d'une économie de services impliquant un couplage d'activités entre producteur et usager, les activités humaines apparaissent à la fois comme processus et comme produit, et tout se passe comme si comprendre leur exercice en vue de leur développement devenait une nouvelle ressource économique et sociale, une nouvelle 'poche' de productivité et donc de rentabilité.

On notera aussi toute l'importance accordée aujourd'hui à la 'transition' qui se focalise sur le changement/les transformations en situation d'incertitude.

\section{En réponse à cette exigence, on constate deux réductions, héritières du paradigme théorie/pratique}

«Il n'est rien pour quoi les Grecs se soient donné une peine aussi obstinée que pour l'éloquence » écrivait Nietzsche (2020).

Une réduction des transformations aux significations portées par le langage ordinaire des acteurs en situation:

Les acteurs, dans leur rapport à leur propre activité, ont une expérience des transformations, et organisent, pensent et disent leur action en termes de transformations. Leur vie sociale et personnelle est une expérience partagée et individuelle de transformations d'eux-mêmes, de leurs activités et interactivités, et de leurs environnements, même si « ces transformations sont silencieuses » (Jullien 2009 ) et si, selon les mots du poète, " nous vivons dans l'oubli de nos métamorphoses » (Eluard, Chagal 1946). La société s'organise à partir de cette expérience partagée.

Lorsque les sujets individuels et collectifs organisent, pensent et disent leur action ils opèrent des constructions de sens et des attributions de significations autour de leur activité : représentations et définitions d'objectifs ou de projets d'action, évaluations etc. Le fondement de cette mentalisation et de cette mise en discours joue probablement une fonction « de sûreté quant aux résultats de l'action » (Gégout 2014 : 59).

On peut alors parler de pensée sociale et/ou personnelle de la transformation, quelquefois qualifiée donc de recherche de sens et de donations de significations par les acteurs.

- Pensée sociale pour les acteurs des champs d'activités (santé, éducation, travail social, économie, politique, culture par exemple etc.) fondés sur des représentations et des conventions sociales sur les transformations auxquels ils sont ordonnés et qui deviennent des 'espaces proposés à l'activité'. 
- Pensée personnelle pour les sujets développant des activités de subjectivation de ces champs, qui deviennent alors des espaces 'investis'.

La reconnaissance par la recherche d'unités fonctionnelles pour les acteurs n'implique pas forcément de se limiter aux catégories subjectives construites par ces acteurs. A propos de chaque type d'action, il est possible de distinguer le langage 'naturel' que tiennent les acteurs sur leurs activités, que l'on peut appeler lexique de l'action, très axiologisé, et un discours se proposant de mettre en objet ce vocabulaire, ces intentions et ces organisations d'activités, que l'on peut appeler lexique d'intelligibilité des actions.

Il est possible dans un discours de recherche sur les actions de faire apparaître à la fois les sens/significations que les acteurs donnent spontanément à leur actes et d'autres significations que celles que qu'ils accordent spontanément. C'est le projet des sciences sociales. Autrement dit on peut à la fois rendre compte de l'activité dans les termes dans lesquels elle se pense, et proposer d'autres catégories que celles dans lesquels elle se pense.

\section{= Une réduction aux constructions académiques}

L'attitude dominante des milieux de recherche est de naturaliser leurs propres construits : les concepts que l'on a créés pour rendre compte de la transformation des entités du monde deviennent des entités du monde. Comme l'indique Ignace Meyerson (1995)

ce n'est pas de ses propres opérations que (notre pensée) est consciente, mais de ses produits (...) C'est donc en même temps une tendance qu'a notre pensée à extérioriser ses créations, ou plus exactement à les considérer comme des réalités extérieures, et dans le cas où cette perspective est la plus poussée, l'objet acquiert une véritable indépendance ; on peut le décrire, on peut apprendre indéfiniment de lui (ibidem : 31).

Axel Honneth parle de réification (2007).

Ce peut être par exemple les schèmes, les habitus, les patterns, le moi, le surmoi, l'inconscient et autres construits de pensée qui finissent par exister aux yeux de ceux qui utilisent ce vocabulaire. Nombre de thèses ou de travaux de recherche se donnent pour objets des concepts, et confondent souvent objet à penser et instrument de pensée.

De façon plus générale, il convient de ne pas confondre les entités du monde et leurs transformations, et les construits pour en rendre compte.

Les fameuses lois de la nature ne 'régissent' pas le monde ; elles sont par contre des outils indispensables dans la construction du rapport que les sujets humains entretiennent avec le monde. L'activité humaine construit ces lois plus qu'elle ne les dévoile. Le monde n'est ni singulier, ni régulier, sinon par rapport à un effort de pensée et d'action. Il est, tout simplement. « Il n'est ni vrai, ni faux, écrit 
Jaspers (1958), c'est seulement notre connaissance qui peut l'être ». Pour François Jullien (2009)

(...) notre intelligence morcelle, isole et stabilise (...) car pour retrouver la vivante perception du changement, il faudrait d'abord se représenter tout changement, tout mouvement, comme indivisible ; or nous appréhendons l'un comme l'autre, le mouvement et le changement, en les décomposant en positions successives et laissons échapper le passage dans lequel se franchit l'intervalle (...) (ibidem : 44-45).

\section{Penser transformation}

Penser transformation est davantage un choix épistémologique qu'un choix théorique, notamment pour cinq raisons :

- Penser est aussi une action. Plus précisément une action de transformation par le sujet qui pense et pour son usage de ses propres représentations. Se représenter est une activité lui permettant de rendre présent à lui-même et de façon différée des objets relevant d'autres espaces d'activité dans lesquels il est également engagé. Transformations pensées et pensées sur les transformations ne relèvent pas des mêmes espaces.

- L'action de pensée peut être considérée elle-même comme 'en transformation' en rapport avec les enjeux des actions de pensée. A rebours du paradigme théorie/pratique, quel que soit le pôle que ce paradigme privilégie, action de pensée et action pensée sont en transformation conjointe, en situation.

- Les transformations sont des outils mentaux et discursifs investis dans l'action de penser et de dire l'activité. Elles assurent aux acteurs et sujet qui pensent les transformations le sentiment de leur possible maîtrise de ces transformations ; de ce point de vue il n'y a pas de différence, d'un point de vue anthropologique, entre les fonctions du discours mythique et du discours à intention scientifique.

- Toutes les actions et activités peuvent être pensées en termes de transformations à partir d'une spécification des fonctions qu'elles jouent pour les acteurs qui les engagent et les performent. Mais en même temps, il convient de penser les nombreuses transformations simultanées et solidaires qui sont également présentes dans la situation.

- Les transformations peuvent être conçues de façons très diverses : par exemple comme des passages d'un état à un autre état, ou comme intervenant sur des processus déjà en cours. Dans certains cas on pourra parler de transformations de transformations.

Toute l'œuvre de François Jullien illustre cette diversité.

- Les concepts construits pour rendre compte des transformations du monde relèvent de ce qu'Einstein désignait comme le monde de l'intelligible. Ne pas confondre avec l'intelligibilité du monde. 


\section{Bibliographie}

Astier P. (2003) La fonction situante de l'activité " Recherche et Formation », $\mathrm{n}^{\circ} 42$ : 87-98, https://www.persee.fr/doc/refor_0988-1824_2003_num_42_1_1828

Barbier J.-M. (1991) Elaboration de projet et planification, Paris, Presses Universitaires de France.

Barbier J.-M. (2017) Vocabulaire d'analyse des activités. Penser les conceptualisations ordinaires, Paris, Presses Universitaires de France.

Barbier J.-M., Durand M. (dir.) (2017) Encyclopédie d'analyse des activités, Paris, Presses Universitaires de France.

Bertaux. D. (2015) Le care comme partie émergée de la production de la vie, « Revue des Sciences Sociales», n 52 : 118-128, https://journals.openedition.org/revss/3257

Berthoz A. (2008) Physiologie de la perception et de l'action, " Annuaire du Collège de France », n $108:$ 303-327, https://journals.openedition.org/annuaire-cdf/314

Canguilhelm G. (1966) Le normal et le pathologique, Paris, Presses Universitaires de France.

Clot Y. (2006) La fonction psychologique du travail, Paris, Presses Universitaires de France.

Dewey J. (1993) Logique. La théorie de l'enquête, Paris, Presses Universitaires de France.

Dubosq J., Clot Y. (2010) L'autoconfrontation croisée comme instrument d'action au travers du dialogue : objets, adresses et gestes renouvelés, « Revue d'anthropologie des connaissances », vol. 4, n $2: 255-286$, https://www.cairn.info/revue-anthropologiedes-connaissances-2010-2-page-255.htm

Dutoit M. coord. (2018) Apprendre d'une expérience rare, Paris, L’Harmattan.

Eluard P., Chagal M. (1946) Le dur désir de durer, Paris, Éditions Arnold Bordas.

Fleury C. (2018) Les irremplaçables, Paris, Gallimard.

Freud S. (1985 [1937]) L'analyse avec fin et l'analyse sans fin, traduction collective in : S. Freud, Résultats, idées, problèmes, tome II, Paris, Presses Universitaires de France.

Gégout P. (2014) John Dewey La quête de certitude, "Recherches \& éducations » [En ligne], 14, http://journals.openedition.org/rechercheseducations/2464, https:// doi.org/10.4000/rechercheseducations 
Honneth A. (2007) La réification. Petit traité de théorie critique, trad. S. Haber, Paris, Gallimard.

James W. (2005) Essais d'empirisme radical, trad. M. Girel, Paris, Agone.

Jullien F. (2009) Les transformations silencieuses, Paris, Grasset.

Lojkine J. (2018) L'anthroponomie de Paul Boccara , "La Pensée », n 1/393 : 79-90, https://www.cairn.info/revue-la-pensee-2018-1-page-79.htm

Malaterrre Ch. (2010) Les origines de la vie. Émergence ou explication réductive, Paris, Hermann.

Meyerson I. (1995) Les fonctions psychologiques et les œuvres, Paris, Albin-Michel.

Nieztsche (2020) Rhétorique, Écrits philologiques, t. 10, trad. A. Merker, présentation A. Merker, Paris, Les Belles Lettres.

Pagès M. (1977) Le travail amoureux. Eloge d'incertitude, Paris, Éditions Bordas.

Simondon G. (2007), Lindividuation psychique et collective : à la lumière des notions de forme, information, potentiel et métastabilité, Paris, Éditions Aubier.

Tap P. (1987) Identité, style personnel et transformation des rôles sociaux, « Bulletin de Psychologie », t. XL, n 379 : 399-403, http://www.pierretap.com/pdfs/71.pdf

Theureau J. (2010) La constitution des savoirs dans l'action, «Intellectica. Revue de l'Association pour la Recherche Cognitive", n 53-54/1-2. Philosophie, Technologie et Cognition : 95-127, www.persee.fr/doc/intel_0769-4113_2010_num_53_1_1180 ; https://doi.org/10.3406/intel.2010.1180

Vygotski L. (2019) Pensée et langage, trad. F. Sève, présentation J. Piaget, Y. Clot, L. Sève, Paris, Éditeur La Dispute.

Weill-Fassina A. (2016) L'image opérative de Dimitri Ochanine en contexte, «Perspectives interdisciplinaires sur le travail et la santé » [En ligne], $\mathrm{n}^{\circ}$ 1/18 : 1-30, http://journals.openedition.org/pistes/4655, https://doi.org/10.4000/pistes.4655

\section{Sources d'Internet}

Brossard M. (2004) Vygotsky Lectures et perspectives de recherches en éducation, https://books.openedition.org/septentrion/14163?lang=fr [consulté : 5.04.2021].

Dutoit M., Barbier J.-M. (2018) Ni auto-formation, ni hétéro-formation : le concept d'apprentissage conjoint, "The Conversation », 1 November, https://theconversation 
.com/ni-auto-formation-ni-hetero-formation-le-concept-dapprentissage-conjoint103651 [consulté : 5.04.2021].

Jaspers K. (1958) Von der Wahrheit. Munich, R. Piper, https://www.degruyter.com/ view/book/9783112312643/10.1515/9783112312643-008.xml [consulté : 5.04.2021].

\section{Professional Action and "Transformation Thinking"}

\section{Summary}

The international trend towards professionalization "through" and "in" higher education has accelerated the emergence of a process of professional education that is not content with preparation of the premises for the anticipated professional activity, but simply prepares for the activity. In particular, professional thinking, which both applies to transformation processes and is one itself.

Developing the concept of "transformation thinking" in this article, we discuss the many dimensions of this "thinking". We analyse, among other things:

- thinking about influencing another perceived as influencing his activity;

- thinking about influencing the activity of another, understood in terms of the mutual connection (couplage) between the acting subject and the activity of the subject, towards which the influence is directed;

- thinking that influencing the other person is also influencing the previous dynamics of the activity of the subject towards which we are acting;

- thinking that influencing the activity of another is constructing a situation of action;

- influencing the activity of another is also a transformation of one's behavior in the course of action;

- influencing the activity of the other person is also a readiness to distinguish intentional transformations and transformations carried out/realized;

- involvement in research on one's professional conduct is also a transformation of oneself.

\section{Działanie profesjonalne i koncept „myśląc transformacje”}

\section{Streszczenie}

Międzynarodowe tendencje w kierunku profesjonalizacji „poprzez” i „w” nauczaniu na poziomie wyższym przyspieszyły pojawienie się procesu kształcenia profesjonalnego, które nie zadowala się przygotowaniem przesłane do przewidywanego działania profesjonalnego, ale wprost przygotowuje do tego działania. W szczególności zaś do myślenia profesjonalnego, które odnosi się zarówno do procesów transformacji, jak też samo nią jest. 
Rozwijając w tym artykule koncept „transfromacje”, omawiamy liczne wymiary tego „myślenia”. Analizujemy m.in.:

- myślenie o odziaływaniu na drugiego ujmowane jako oddziaływanie na jego aktywność;

- myślenie o oddziaływaniu na aktywność drugiego ujmowane jest w kategoriach wzajemnego powiązania (couplage) między podmiotem działającym i aktywnością podmiotu, wobec którego skierowane jest oddziaływanie;

- myślenie o tym, że oddziaływanie na drugiego jest również oddziaływaniem na uprzednią dynamikę aktywności podmiotu, wobec którego oddziałujemy;

- myślenie o tym, że oddziaływanie na aktywność drugiego stanowi konstruowanie sytuacji działania;

- oddziaływanie na aktywność drugiego jest także transformacją swego postępowania w trakcie działania;

- oddziaływanie na aktywność drugiego jest też gotowością na wyodrębnienie transformacji intencjonalnych i transformacji dokonanych/zrealizowanych;

- zaangażowanie $\mathrm{w}$ badania nad swym postępowaniem profesjonalnym jest także transformacją siebie.

\section{Pour citer cet article}

Barbier J.-M. (2021) Action professionnelle et pensée transformation, „Nauki o Wychowaniu. Studia Interdyscycplinarne" 2(13), 25-47, https://doi.org/10.18778/24504491.13.04 